\title{
Vasoaktives Intestinales Polypeptid (VIP) im Atemtrakt: Physiologie und Pathophysiologie
}

\author{
D. A. Groneberg' \\ K. F. Rabe ${ }^{2}$ \\ U. Wagner ${ }^{3}$ \\ A. Fischer ${ }^{1}$
}

\section{Vasoactive Intestinal Polypeptide in the Respiratory Tract: \\ Physiology and Pathophysiology}

\section{Zusammenfassung}

Durch die Charakterisierung verschiedenster pulmonaler Effekte gewannen peptiderge Neuromediatoren in den letzten Jahren zunehmend an Bedeutung für das Verständnis physiologischer und pathophysiologischer Mechanismen im Atemtrakt. Dabei spielt Vasoaktives Intestinales Polypeptid (VIP) eine besondere Rolle aufgrund seiner potenziell anti-inflammatorischen und immunmodulierenden Wirkung. Es wird neurophysiologisch zu den Mediatoren des inhibierenden nicht-adrenergen nicht-cholinergen Nervensystems (i-NANC) gezählt. In den menschlichen Atemwegen sind VIP-haltige Nervenfasern in der glatten Muskulatur von Trachea und Bronchien sowie von Pulmonalgefäßen vorhanden. Neben starken vasodilatorischen Eigenschaften besitzt VIP auch eine hohe bronchodilatorische Potenz. Es gibt eine Vielzahl pulmonaler Erkrankungen bei denen VIP pathophysiologisch beteiligt sein könnte. In dieser Hinsicht konnten veränderte VIP-Spiegel bei entzündlichen Erkrankungen der oberen und unteren Atemwege sowie bei der primären pulmonalen Hypertonie nachgewiesen werden. Aufgrund der schnellen enzymatischen Inaktivierung konnte eine auf VIP basierende Therapie bis jetzt noch nicht zum klinischen Einsatz gebracht werden, obwohl VIP starke bronchodilatorische Eigenschaften besitzt. Auch konnte der Einsatz synthetischer VIP-Agonisten keine Therapieverbesserung im Vergleich zur herkömmlichen Asthmatherapie bieten. Diesen Befunden stehen tierexperimentelle Daten

\section{Abstract}

Peptidergic neuromediators have gained importance in the field of respiratory physiology and pathophysiology due to the characterisation of numerous pulmonary effects in the past years. With regard to the multitude of mediators, the neuropeptide vasoactive intestinale polypeptide (VIP) plays a special role as it exerts potent anti-inflammatory and immunomodulatory effects. Neurophysiologically the peptide has been attributed to the family of the inhibitory non-adrenergic non-cholinergic (i-NANC) neuromediators of the pulmonary innervation. VIPcontaining nerve fibres are localized in the airway and vascular smooth muscle layers of trachea and bronchi in the human respiratory tract. Apart from strong vasodilatory effects, the peptide also shows a high bronchodilatory potency. In a large number of respiratory diseases VIP may play a pathophysiological role. In this respect, increased levels of VIP have been demonstrated for inflammatory diseases of the upper and lower airways and the peptide may also play a role in pulmonary hypertension. Due to its fast enzymatic cleavage, VIP-based therapies have not been used in routine therapy so far. Also, the use of synthetic VIP-agonists did not lead to an improved outcome in patients with bronchial asthma if compared to classical drugs. However, recent data from animal experiments indicate potent immunomodulatory effects which suggest a future use of this mediator and its agonists in the therapy of immune diseases.

Institutsangaben

${ }^{1}$ Klinische Forschergruppe Allergologie, Charité - Universitätsmedizin Berlin, Freie Universität Berlin \& Humboldt-Universität zu Berlin, 13353 Berlin (Leiter: Prof. Dr. A. Fischer)

${ }^{2}$ Abteilung für Lungenheilkunde, Universitätsklinikum Leiden, NL-2300 RC Leiden, Niederlande (Leiter: Prof. Dr. K. F. Rabe)

${ }^{3}$ Medizinische Klinik, Abteilung für Lungenheilkunde, Universitätsklinikum, Baldingerstraße 1, 35043 Marburg (Leiter: Prof. Dr. C. Vogelmeier)

Korrespondenzadresse

Dr. med. David A. Groneberg - Klinische Forschergruppe Allergologie, Charité - Universitätsmedizin Berlin, Freie Universität Berlin \& Humboldt-Universität zu Berlin · Augustenburger Platz 1 .

13353 Berlin · E-mail: david.groneberg@charite.de

Eingang: 17. November 2003 - Nach Revision akzeptiert: 31. Januar 2004

Bibliografie

Pneumologie 2004; 58: 330-338 • @ Georg Thieme Verlag KG Stuttgart · New York

DOI $10.1055 / \mathrm{s}-2004-818352$

ISSN 0934-8387 
zur Immunmodulation durch VIP gegenüber. Sie weisen auf einen zukünftigen Einsatz des Mediators und seiner Agonisten bei der Behandlung immunologischer Erkrankungen hin.

\section{Einleitung}

In Anbetracht der weitreichenden Entwicklungen der neuro-immunologischen Forschung hat sich die Frage, ob Asthma bronchiale eine immunologische oder wie früher vermutet eine neuronale Erkrankung ist [1], darin aufgelöst, dass es sich bei diesem Erkrankungsbild primär um eine komplexe entzündliche Krankheitsentität handelt, wobei jedoch auch wichtige Wechselwirkungen des Immunsystems mit der Atemwegsinnervation existieren. Die den entzündlichen Veränderungen zugrunde liegenden Mechanismen werden dabei von einer Vielzahl an Mediatoren beeinflusst. Im Bereich der Pathophysiologie und -biochemie des Asthma bronchiale sind mittlerweile bereits über fünfzig Mediatoren mit Effekten auf verschiedenste pulmonale Funktionen beschrieben worden [2]. Fortschritte in diesem Gebiet wurden vor allem durch die Entwicklung neuer, potenter Inhibitoren gemacht, die entweder die Rezeptoren der Mediatoren blockieren oder sie selbst inhibieren [3-6]. Der Syntheseort der einzelnen Mediatoren liegt sowohl im Bereich von Entzündungszellen wie Mastzellen, Eosinophile, Basophile, Neutrophile oder T-Lymphozyten, als auch im Bereich gewebsständiger Zellen wie Epithelzellen, Endothelzellen, Myozyten oder Atemwegsneuronen [7].

Die als neurogene Entzündung beschriebene Komponente bewirkt durch die lokale Freisetzung peptiderger Mediatoren unter anderem klassische Entzündungsmerkmale wie „Calor“, „Rubor“ und „Dolor“ [8].

Neben den klassischen Mediatoren Noradrenalin in postganglionären sympathischen und Acetylcholin in parasympathischen Nervenfasern, existiert eine Reihe von peptidergen Mediatoren, die ausgeprägte funktionelle Effekte auf verschiedenste respiratorische Funktionen wie den Muskeltonus der Gefäße und Atemwege, die Drüsensekretion und auf Entzündungs- und Immunzellen haben [9]. Die Neuropeptide gehören zu keinem morphologischen eingrenzbaren Nervensystem innerhalb der Atemwege und ihre Effekte wurden deshalb unter dem Begriff des nichtadrenergen nicht-cholinergen (NANC)-System zusammengefasst [10]. Aufgrund physiologischer und pharmakologischer Erkenntnisse wurden die NANC-Mediatoren in die zwei funktionell divergenten Gruppen des exzitatorischen NANC-Systems (e-NANC) und des inhibitorischen NANC-Systems (i-NANC) eingeordnet [11].

\section{Anti-inflammatorische peptiderge Mediatoren}

Im Gegensatz zu den Mediatoren des exzitatorischen NANC-Systems, welchen in der jüngeren Vergangenheit ein aggravierende Rolle bei der Entstehung und Fortführung allergischer Erkrankungen zugeordnet wurde [12,13], spiegeln andererseits die zum inhibitorischen NANC-System (i-NANC) gehörenden Media- toren eine wesentlich inhomogenere Gruppe wider, deren Einflüsse bei der neurogenen Entzündung teilweise noch ungeklärt sind. Zu den i-NANC Mediatoren gehören Vasoaktives Intestinales Polypeptid (VIP) [14], Neuropeptid Y (NPY) [15], das gasförmige Stickstoffmonoxid (NO) [16] oder auch endogene Opioide [17].

Unter der Vielzahl der potenziell anti-inflammatorischen Mediatoren spielt der 1969 von Said und Mutt identifizierte Mediator VIP eine besondere Rolle aufgrund zahlreicher jüngster tierexperimenteller Hinweise bezüglich immunmodulierender Effekte [18] und seiner starken Expression in Organen wie dem Atemtrakt [14] oder der Haut [19]. Auf der Basis der Expression von VIP und seiner Bindungsstellen innerhalb des Atemtrakts wurde in den vergangenen Jahren eine Vielzahl pulmonaler Effekte beschrieben (Abb.1).

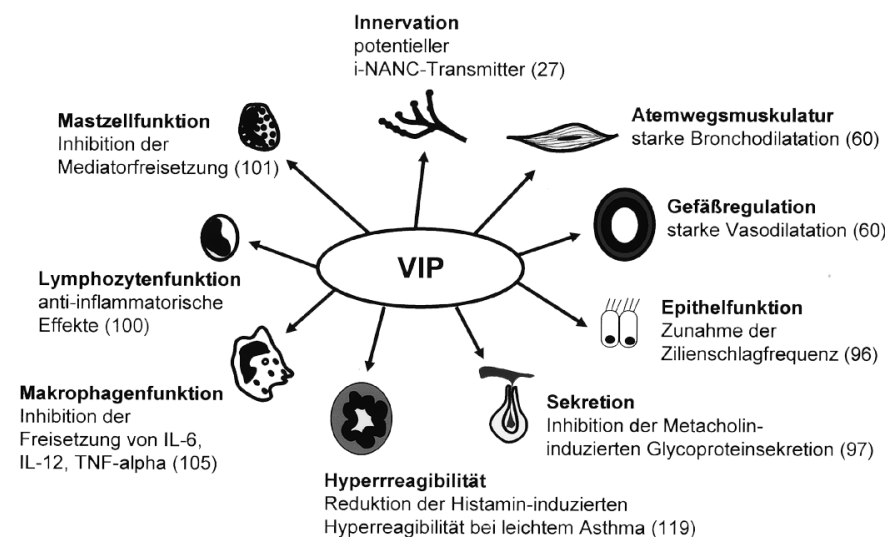

Abb. 1 Effekte von VIP im Atemtrakt. VIP-positive Nervenfasern ziehen zu einer Vielzahl pulmonaler Effektorzellen und beeinflussen deren Funktion über direkte und indirekte Signalwege. Darüber hinaus gibt es Wechselwirkungen mit verschiedenen Zellen des unspezifischen und spezifischen Immunsystems.

\section{Struktur und Lokalisation}

Das 28 Aminosäuren umfassende Polypeptid VIP wurde erstmals aus dem Duodenum aufgrund seiner vasodilatorischen Effekte isoliert [20-22]. Das Gen des Mediators ist auf Chromosom 6q24 lokalisiert und kodiert Pro-VIP, welches ebenfalls die Sequenz des verwandten Mediators Peptide-Having-Carboxyterminal-Methionine (PHM-27) enthält [23]. Zusammen mit anderen Peptiden wie „Pituitary Adenylate Cyclase Activating Polypeptide“ (PACAP), „Peptide Having Carboxy-terminal Methionine/Isoleucine“ (PHM/PHI), Peptide Histidine Valine (PHV), Sekretin, Glukagon, Growth hormone-releasing factor (GRF) und Helodermin bildet VIP eine Familie von Peptiden, die aufgrund ähnlicher Strukturen durch eine Gemeinsamkeit verschiedener biologischer Effekte gekennzeichnet ist [24].

VIP kann durch Enzyme wie Neutrale Endopeptidase (NEP) oder Mastzell-Tryptase inaktiviert werden, wobei humanes VIP hauptsächlich durch NEP zu inaktiven Metaboliten prozessiert wird $[25,26]$ (Abb. 2).

VIP-positive Nervenfasern konnten in den humanen Atemwegen im Bereich der glatten Muskulatur von Atemwegen und Gefäßen, 

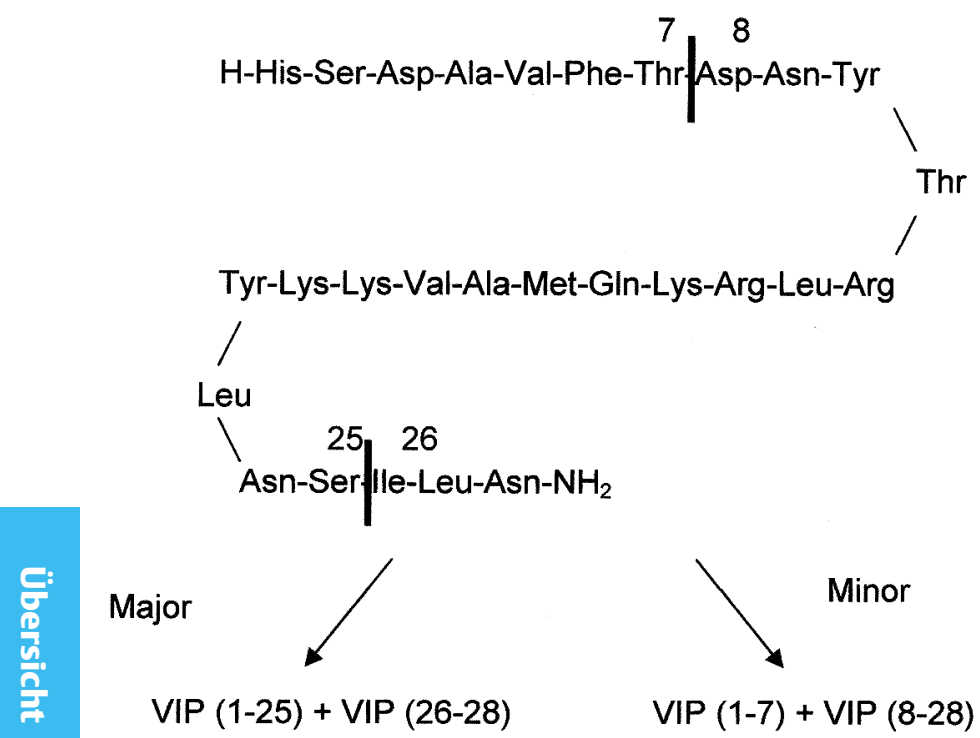

Major

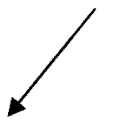

VIP (1-25) + VIP (26-28)

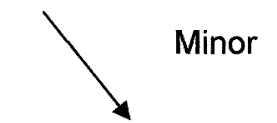

VIP (1-7) + VIP (8-28)
Abb. 2 Enzymatische Inaktivierung von humanem VIP in den Atemwegen durch Peptidasen. Die Hauptstelle (Major) der enzymatischen Inaktivierung des 28-Aminosäuren Peptids VIP liegt bei Position $\mathrm{Ser}^{25}-1 \mathrm{e}^{26}$, während eine sekundäre Inaktivierungsstelle (Minor) bei der Position Thr ${ }^{7}-$ Asp $^{8}$ vorhanden ist.

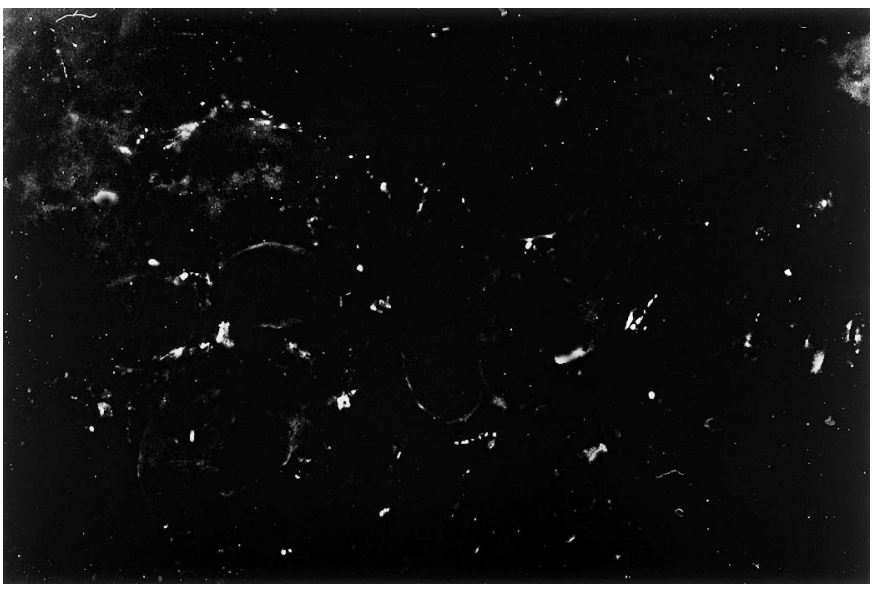

Abb. 3 Dichte Innervation der Drüsen durch VIP-positive Nervenfasern in der humanen Trachea. Immunhistochemische Darstellung mittels eines gegen VIP gerichteten Primärantikörpers und eines fluoreszierenden Sekundärantikörpers. Das VIP-spezifische immunhistochemische Signal lässt sich in submukös gelegenen Nervenfasern erkennen, die zu Drüsenarealen ziehen. Originalvergrößerung $\times 250$.

rund um Drüsen (Abb. 3) und in der Lamina propria nachgewiesen werden, wobei die Faserdichte mit der Größe der Atemwege abnimmt $[27,28]$. VIP wird je nach Spezies mit verschiedenen anderen Mediatoren koexprimiert. So wurde es beispielsweise im Atemtrakt des Meerschweinchens zusammen sowohl in sympathischen [29] als auch parasympathischen [30] Nervenfasern der Atemwege nachgewiesen.

\section{VIP-Rezeptoren}

Nach dem in der Vergangenheit autoradiographische Bindungsstudien zur Lokalisation von VIP-Rezeptoren herangezogen wurden [31], konnte durch die Klonierung zweier unterschiedlicher

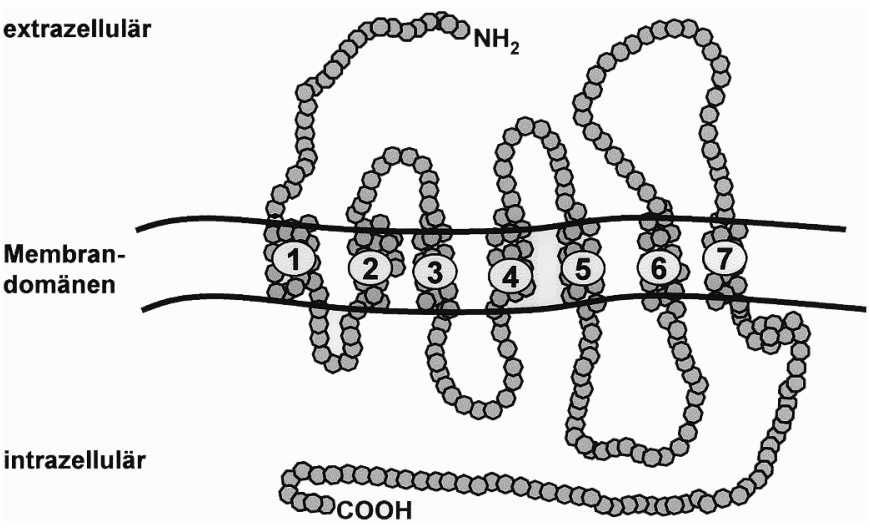

Abb. 4 Struktur der VIP-Rezeptoren. Bei beiden Proteinen handelt es sich um G-Protein gekoppelte Membranenproteine mit 7 Transmembrandomänen und einem intrazellulär gelegenen carboxyterminalen Ende.

Rezeptoren eine molekularbiologische Grundlage für die vielfältigen Wirkungsmechanismen des Mediators VIP gefunden werden. Dabei handelt es sich bei den VIP-Rezeptoren um 2 G-Protein-gekoppelte Rezeptoren mit 7 Transmembrandomänen (Abb. 4).

\section{VPAC1-Rezeptor}

Der VPAC1-Rezeptor war ursprünglich als einziger VIP-Rezeptor beschrieben [32]. Später wurde er umbenannt in VIP ${ }_{1}$-Rezeptor [33], VIP/PACAP Typ-II-Rezeptor [34] und PVR 2 [35]. In der jüngsten Nomenklatur der Internationalen Union der Pharmakologie wurde der Rezeptor als $\mathrm{VPAC}_{1}$-Rezeptor terminiert [36]. Er wurde erstmals aus der Rattenlunge [32] und später auch aus humanen Geweben isoliert [37,38]. Es sind bis zum heutigen Zeitpunkt keine Splicevarianten bekannt. Demgegenüber existieren signifikante Spezies-spezifische Unterschiede in der Pharmakologie des Rezeptors [39]. Bis jetzt konnten mehrere VPAC $_{1}$-Rezeptoragonisten beschrieben werden: Das VIP/GRF-Hybrid $\left[\operatorname{Lys}^{15}, \operatorname{Arg}^{16}\right.$, Leu $\left.^{27}\right] \mathrm{VIP}(1-7) \operatorname{GRF}(8-27)-\mathrm{NH}_{2}$ stellt einen selektiven $\mathrm{VPAC}_{1}$-Rezeptoragonisten dar, der Growth hormone-releasing factor (GRF)-Rezeptoren nicht aktiviert [40]. [Arg $\left.{ }^{16}\right]-S e-$ kretin ist ein Agonist von $\mathrm{VPAC}_{1}$ - und Sekretinrezeptoren. [Acetyl-His ${ }^{1}$, D-Phe ${ }^{2}$, Lys $^{15}$, Arg $^{16}$ ] VIP (3-7)GRF(8-27)- $\mathrm{NH}_{2}$ (PG 97-269) ist ein selektiver Antagonist von $\mathrm{VPAC}_{1}$-Rezeptoren [41].

\section{VPAC2-Rezeptor}

Ein zweiter, vormals als $\mathrm{VIP}_{2}$-Rezeptor [33], PACAPR-3 [42], oder PVR3 [35] benannter Rezeptor wurde in der letzten Nomenklatur als VPAC - -Rezeptor benannt [36]. Dieser Rezeptor bindet sowohl VIP als auch PACAP mit einer gleichwertigen Affinität und wurde in Geweben von Ratte [33,43], Maus [42] und Mensch identifiziert $[44,45]$. Auch bei diesem Rezeptor sind bislang keine Splicevarianten identifiziert worden. In Zelllinien exprimiert, bindet der Rezeptor VIP, PACAP-38, PACAP-27, sowie PHV und PHI. Demgegenüber besteht nur eine sehr geringe Affinität gegenüber GRF und Sekretin. Es gibt hochselektive $\mathrm{VPAC}_{2}$-Agonisten, die von ihrer Struktur zyklische Peptide sind. Neben Ro 25-1553 [46], welches zuerst als ein anti-inflammatorisches bronchorelaxierendes Medikament entwickelt wurde [47-49], existiert Ro 25 - 1392 [50]. 
Seit der Identifizierung von VIP-Rezeptoren in den neunziger Jahren [36] und der Etablierung kombiniert molekularbiologisch-morphologischer Methoden wie der nicht-radioaktiven in situ-Hybridisierung [51] standen Techniken zur Verfügung, die es ermöglichten Rezeptoren für VIP auf der transkriptionellen Ebene nachzuweisen. So wurde durch den Einsatz VPAC2-spezifischer Sonden gezeigt, dass VPAC2-mRNA in basalen sowie zilientragenden Atemwegsepithelzellen von Trachea und extrasowie intrapulmonalen Bronchien vorhanden ist [52]. Diese Ergebnisse zeigten, dass zilientragende Epithelzellen das morphologische Korrelat der früher berichteten Bindung von radioaktiv markiertem VIP im Bereich des Atemwegsepithels [31] bezüglich VPAC2 darstellen, wohingegen Becherzellen keine VPAC2-mRNA exprimieren. Darüber hinaus wurden ebenfalls keine Signale in Arealen der glatten Atemwegsmuskulatur sowie in der Gefäßmuskulatur gefunden. Diese Befunde widersprechen früher gemachten Beobachtungen [31]. Da auch moderne Arbeiten mittels Rezeptor-spezifischen Antikörpern VPAC2-Protein im Bereich der glatten Muskulatur von Rattenatemwegen nachweisen konnten [53], stehen wahrscheinlich nicht genügend VPAC2-mRNA Kopien in den Myozyten der Atemwegsmuskulatur zur Verfügung, um eine Detektion durch in situ-Hybridisierung zu ermöglichen. Zukünftige Studien, die sich hochsensitiver Verfahren zur Lokalisation von mRNA wie der Lasermikrodissektions-gestützten RT-PCR [54] bedienen, werden diese kontroversen Ergebnisse auflösen können.

Im Bereich der submukösen Drüsen wiesen sowohl muköse als auch seröse Drüsenabschnitte VPAC2-mRNA auf [52]. Dabei gab es keine Unterschiede zwischen den einzelnen Abschnitten der Trachea, extra- sowie intrapulmonaler Atemwege, so dass VPAC2 an den Effekten von VIP auf die Drüsensekretion beteiligt zu sein scheint. Im Bereich von Bindegewebe und Knorpel wurde in Einklang mit früheren Studien keine VPAC2-mRNA gefunden $[31,55]$.

In der peripheren Lungen zeigte sich VPAC2-mRNA in Alveolarmakrophagen. Ebenfalls wurde VPAC2-mRNA in peribronchialen Immunzellen gefunden [52]. Diese morphologischen Ergebnisse weisen auf eine Rolle von VIP in der lokalen Modulation von Immunreaktionen hin, die durch jüngste Studien mit VPAC2-Gendepletierten und VPAC2-transgenen Mäusen auf der tierexperimentellen Ebene hervorgehoben werden konnte $[18,56]$.

\section{Biologische Funktionen im Atemtrakt}

\section{Atemwegsmuskulatur}

VIP besitzt starke bronchodilatorische Eigenschaften in vivo und in vitro. Mit einer fast einhundertfach erhöhten bronchodilatorischen Potenz gegenüber Isoproterenol ist VIP der stärkste endogene Bronchodilator [57], wobei der Ort der maximalen Wirkung hauptsächlich im Bereich der zentralen Atemwege anzusiedeln ist. Die Bronchodilatation ist unabhängig von adrenergen oder cholinergen Rezeptoren oder Cyclooxygenasen [58-60]. Im Gegensatz zu Isoproterenol beziehen sich die Effekte von VIP eher auf die Resistance als auf die dynamische Compliance [61]. In dieser Hinsicht konnte ebenfalls gezeigt werden, dass VIP eine größenabhängige Wirkung zeigt, die parallel zu der Größe der Atemwege abnimmt [58]. Diese Wirkungsabnahme ist konsis- tent mit der Verteilung von VIP-positiven Nervenfasern, die in den peripheren Atemwegen ebenfalls abnimmt [27]. So konnte auch autoradiographisch eine Abnahme von Bindungsstellen nachgewiesen werden [31].

Trotz der in vitro nachgewiesenen starken bronchodilatorischen Effekte von VIP in humanen Atemwegen, welche die Effekte anderer konstriktorischer Mediatoren wie Histamin, Prostaglandin $\mathrm{F}_{2 \alpha}$, Endothelin, Leukotrien $\mathrm{D}_{4}$, Kallikrein und NKA signifikant inhibieren [62,63], konnte der Mediator aufgrund seiner starken vasodilatorischen Potenz nicht systemisch im klinischen Bereich eingesetzt werden.

Die inhalative Gabe von VIP führte trotz seiner Wirkung gegenüber der Histamin- und Prostaglandin $\mathrm{F}_{2 \alpha}$-induzierten Bronchokonstriktion in Kaninchen [64] zu keinem signifikantem Effekt bei Patienten mit einem Belastungsasthma [65]. Ebenso zeigten sich nur geringe Effekte bei der Histamin-induzierten Bronchokonstriktion beim Menschen [66]. Diese unerwarteten schwachen Effekte nach inhalativer VIP-Gabe können durch eine mangelnde Penetration des Peptids durch das Bronchialepithel sowie durch eine schnelle Inaktivierung durch epitheliale Peptidasen erklärt werden [66,67]. Aufgrund seiner Größe von 28 Aminosäuren kann der Mediator dabei nicht von Peptidtransportern wie PEPT1 und PEPT2 transportiert werden, die in den Atemwegen [68 - 70] und dem peripheren Nervensystem [71] exprimiert werden.

Bezüglich einer mangelhaften Penetration nach inhalativer Gabe führte die Denudierung des Epithels zu einer Verstärkung der VIP-induzierten Relaxation von Trachealsegmenten [72]. Es zeigten auch Peptidase-resistente VIP-Analoga stärkere Effekte $[73,74]$. In einer neueren Studie konnte in dieser Hinsicht unter Verwendung des selektiven VPAC2-Rezeptoragonisten Ro 25-1553 in vitro eine starke dilatorische Potenz des Agonisten in der humanen Bronchialmuskulatur und in Pulmonalarterien bewiesen werden [75].

\section{Gefäßregulation}

VIP wurde ursprünglich aufgrund seiner vasodilatorischen Eigenschaften identifiziert und gehört auch in den Atemwegen zu den stärksten endogenen Vasodilatoren. Dabei relaxiert es potent Gefäße in den oberen Atemwegen [76,77], Trachea, Bronchien [78] sowie die Pulmonalarterien [62,79-81]. Bezüglich der Stärke seiner Effekte konnte gezeigt werden, dass die VIP-induzierte Vasodilatation stärker in der trachealen als in der bronchialen Zirkulation ist [82]. Darüber hinaus ist der vasodilatorische Effekt von VIP ca. zweihundertfach stärker als der von Prostazyklin [60] und unabhängig von der Integrität des Endothels $[83,84]$.

\section{Sekretion}

Ein weiterer pathophysiologischer Mechanismus, der zu einer wesentlichen Verschlechterung der Atemfunktion bei schwerem Asthma bronchiale führen kann, ist die Mukushypersekretion. Molekulare Grundlage des in den oberen [85] und unteren Atemwegen [86] gebildeten Mukus sind Glykoproteine, die auch als Muzine bezeichnet werden. Im Falle des fatalen Status asthmaticus können diese Proteine aufgrund einer massiven reflexartigen 
Sekretion zu der Verlegung der Atemwege und zum Tode führen $[87,88]$.

Im Gegensatz zu der klaren Datenlage bezüglich der bronchound vasodilatorischen Wirkung von VIP sind dessen regulatorische Effekte bezüglich der Mukussekretion weitestgehend kontrovers. Es gibt ein enges Netzwerk VIP-positiver Nervenfasern im Bereich der Drüsen [89], so dass die Partizipation von VIP in der Regulation der Drüsenaktivität nahe liegt. Demgegenüber wurden bis jetzt eine Reihe widersprüchlicher Ergebnisse zum Einfluss von VIP auf die Sekretion publiziert: Auf der einen Seite stehen Befunde, die eine Stimulation der Mukussekretion durch VIP in Frettchentrachealdrüsen [90] oder Rattentrachealzellen [91] in vitro nachgewiesen haben. Andererseits liegen Arbeiten vor, die eine Inhibition der cholinerg-stimulierten Sekretion durch VIP in der Frettchentrachea in vitro belegen konnten [92]. In der Trachea von Katzen wurde wiederum die cholinerg-stimulierte Sekretion durch VIP in vitro gefördert [93]. Für die Hundetrachea konnte schließlich gezeigt werden, dass VIP die aktive Sekretion von Chlorid-Ionen in vitro stimuliert [94]. Auch führte die Kombination von VIP mit anderen sekretorischen Agonisten zu Veränderungen in der Schleimsekretion. So wurde eine potente VIP-stimulierte Induktion der sekretorischen Antwort auf Phenylephrine [95], sowie eine VIP-induzierte Zunahme der Zilienschlagfrequenz in kultivierten Kaninchentrachealepithelzellen beschrieben, die durch Zugabe eines VIP-Antagonisten aufgehoben wurde [96].

Im Gegensatz zu den Befunden in Tiermodellen, die teilweise eine stimulierende Eigenschaft von VIP auf die Mukussekretion postulierten, konnte für die humane Trachea in vitro bis jetzt nur ein inhibitorischer Effekt gegenüber Metacholin-stimulierter Glykoproteinsekretion gefunden werden [97]. Im Bereich der oberen Atemwege konnte für Zellen aus der nasalen Mukosa gezeigt werden, dass VIP die Sekretion von Lactoferrin stimulieren kann, ohne jedoch eine große Wirkung auf Mukusglykoproteinsekretion zu haben [28].

\section{Interaktion mit Zellen des Immunsystems}

Es konnte gezeigt werden, dass eine Vielzahl an Immunzellen wie beispielsweise Eosinophile, Mastzellen oder T-Lymphozyten VIP-Rezeptoren exprimieren und VIP funktionell erkennen $[98,99]$. Dabei sind die wesentlichen immunmodulierenden Effekte von VIP von anti-inflammatorischer, inhibierender Natur und mit einer Reduktion der zellulären Proliferation verbunden [100]. So inhibiert VIP die Freisetzung von Mediatoren pulmonaler Mastzellen [101], interagiert mit T-Lymphozyten [102], verhindert Xanthine-Oxidase-abhängige Gewebsdestruktion [103] und vermittelt die Neutralisierung von Sauerstoffradikalen [104]. Ebenso inhibiert VIP die Produktion von Interleukin (IL)-6, IL-12, Tumor Nekrose Faktor (TNF)- $\alpha$ sowie NO und stimuliert die IL-10-Produktion. Dabei werden die Effekte auf TNF- $\alpha$, IL-10, IL-12 und NO wahrscheinlich auf der transkriptionellen Ebene durch den VPAC1-Rezeptorsubtyp über die Transkriptionsfaktoren NF-kB und CRE vermittelt [105]. Im Bereich der rheumatoiden Arthritis konnte dabei auf der tierexperimentellen Ebene gezeigt werden, dass VIP über VPAC1 stark entzündungshemmend ist [106]. Im Gegensatz zu diesen VPAC1-vermittelten Effekten konnten jüngste Studien ebenfalls darauf hinweisen, dass auch VPAC2 immunmodulatorische anti-inflammatorische Effekte von VIP vermitteln kann. So wurde in einer VPAC2-Gen-depletierten Maus eine Neigung zu Allergien festgestellt [18] und darüber hinaus eine verminderte Expression des VPAC2-Rezeptors in Mastzellen von Patienten mit atopischer Dermatitis [107].

\section{Rolle bei Erkrankungen des Atemtrakts}

\section{Asthma bronchiale}

Die genauen Zusammenhänge der Beteiligung von VIP an pathophysiologischen und pathobiochemischen Mechanismen des Asthma bronchiale sind noch ungeklärt [14]. Eine Hypothese geht davon aus, dass die gestörte zelluläre Vermittlung der Effekte von VIP zu einem erhöhten Tonus der Atemwegsmuskulatur führt. Erste Studien, die eine selektive Verminderung VIP-positiver Nervenfasern bei Patienten mit Asthma bronchiale [108] zeigten, konnten in Folgearbeiten allerdings nicht bestätigt werden [109-111]. Im Asthma-Tiermodell konnte gezeigt werden, dass erhöhte VPAC1- und VPAC2-mRNA Spiegel in Lymphozyten aus bronchoalveolärer Lavage vorhanden sind [112].

Eine Reduktion protektiver Effekte des Neuropeptids ist über einen erhöhten Abbau zu erklären. So sezernieren Entzündungszellen VIP-degradierende Enzyme [113], deren Spiegel (z.B. Mastzell-Tryptase) bei allergischem Asthma bronchiale erhöht sind [114]. Auch wurden VIP-neutralisierende Antikörper im Patientenserum gefunden [115]. Eine klinische Studie bezüglich der Zusammenhänge von VIP-Plasmaspiegeln und körperlicher Belastung bei Kindern mit Asthma zeigte, dass belastungsinduziertes Asthma mit erhöhten VIP-Plasmaspiegeln einhergeht [116]. Im Gegensatz dazu waren bei erhöhten CGRP-, Substanz P- und NPY-Spiegeln die Plasmaspiegel von VIP bei Patienten mit schweren Asthmaattacken verringert [117].

Aufgrund der potenten bronchorelaxierenden und anti-inflammatorischen Eigenschaften galt VIP als ein potenzieller Kandidat für die Entwicklung neuer Asthmatherapeutika. Im Gegensatz zu ersten Studien bei Asthmatikern [67], die eine signifikante Bronchodilatation und Schutz gegenüber Histamin-induzierter Bronchokonstriktion durch VIP nachwiesen, konnten spätere Arbeiten diese protektiven Effekte nicht nachvollziehen. So zeigte beispielsweise die intravenöse VIP-Gabe (1,3 oder $6 \mathrm{pmol} /$ $\mathrm{kg} \cdot \mathrm{min}^{-1}$ ) keinen Effekt auf die Atemfunktion [118], wobei es in hohen Dosen sogar zu Blutdruckabfall und moderater Tachykardie führte. Bei Patienten mit leichtem Asthma reduzierte inhalativ verabreichtes VIP $(100 \mu \mathrm{g})$ die bronchiale Reagibilität gegenüber Histamin [119], währenddessen es bei Patienten mit Belastungsasthma keine wesentliche protektive Wirkung zeigte [65]. Diese schwachen Effekte nach inhalativer VIP-Gabe wurden auf eine mangelnde Penetration des Neuropeptids durch das Atemwegsepithel und seine enzymatische Inaktivierung erklärt $[66,67]$.

In jüngsten Arbeiten mit dem selektiven VPAC2-Rezeptoragonisten Ro 25 - 1553 konnte bei 24 Patienten mit moderatem Asthma nach Inhalation von $600 \mu \mathrm{g}$ Ro 25 - 1553 ein mit Formoterol vergleichbarer bronchodilatorischer Effekt innerhalb von 3 Minuten nachgewiesen werden, der im Gegensatz zu Formoterol (24 h) allerdings nach 5 Stunden nachließ [120]. 


\section{Primäre pulmonale Hypertonie}

Die primäre pulmonale Hypertonie ( $\mathrm{PPH}$ ) ist eine mit progredientem Rechtsherzversagen einhergehende Erkrankung mit einem in der Regel fatalen Ausgang [121]. Die Therapie dieser Erkrankung hat sich in den vergangenen Jahren durch mehrere neue Ansätze enorm verbessert [122]. Dabei kommen neben Prostazyklinen [123] auch Phosphodiesteraseblocker wie Sildenafil [124] in Betracht, wobei beide Therapieansätze sowie deren Kombination in klinischen Studien einen klaren Nutzen zeigten [121,125]. Ein weiterer Therapieansatz basiert auf dem Einsatz von hochdosierten Kalziumantagonisten, die bei einer Untergruppe von Patienten mit PPH zu einer Verbesserung der Lebenserwartung führten [126]. Ebenso können Endothelinrezeptorblocker eingesetzt werden, für die im Fall des nicht selektiven Blockers Bosentan bereits ein signifikanter Therapieerfolg bei Patienten mit PPH aufgezeigt werden konnte. Insgesamt werden voraussichtlich Kombinationstherapien die zukünftige Therapie darstellen, wobei zur Standardisierung internationale multizentrische Studien erforderlich sind.

Aufgrund ihrer extrem starken vasodilatorischen Effekte in der systemischen und pulmonalen Zirkulation können auch VIP bzw. seine synthetischen Agonisten als potenzielle Kandidaten für die Entwicklung neuer PPH-Therapeutika gesehen werden [127]. Vor kurzem konnte nachgewiesen werden, dass die Serumspiegel von VIP und die Anzahl VIP-positiver Nervenfasern in pulmonalen Gefäßen bei Patienten mit PPH vermindert sind, wohingegen VIP-Rezeptoren vermehrt exprimiert werden [128]. Nach dreimonatiger Behandlung mit einer täglichen inhalativen Gabe von VIP in einer Dosis von $200 \mu$ g zeigte sich bei 4 Patienten mit PPH eine Verminderung des mittleren pulmonal-arteriellen Drucks um $13 \mathrm{~mm} \mathrm{Hg}$ von $59 \pm 8$ (S.D.) mmHg auf $46 \pm$ $7 \mathrm{~mm} \mathrm{Hg}(\mathrm{p}<0,01)$. Ebenso besserten sich das Herzminutenvolumen, die 6 Minuten-Gehstrecke sowie der Borg-Index [128]. Auf der Grundlage dieser Daten sollen weitere Studien bezüglich des Nutzens von VIP bei der Behandlung der PPH durchgeführt werden.

\section{Erkrankungen des oberen Atemtrakts}

Auch im Rahmen chronisch-entzündlicher Erkrankungen des oberen Atemtrakts konnten Veränderungen des Profils VIP-positiver Nervenfasern festgestellt werden.

So wurde die irritativ-toxische Rhinitis untersucht, welche durch eine chronische Exposition gegenüber arbeits- und umweltmedizinisch relevanten Noxen wie beispielsweise Ozon, Formaldehyd, Nickel, Chrom, Lösungsmittelinhaltsstoffe und Tabakrauch entstehen kann [129]. Neben NPY-positiven Nervenfasern waren bei dieser Erkrankung ebenfalls VIP-positive Fasern signifikant erhöht, wohingegen die Anzahl Substanz P- und CGRP-positiver Fasern im Normalbereich lag [130]. Im Gegensatz zur toxischen Rhinitis waren bei der hyperreflektorischen Rhinitis neben VIPauch Substanz P-positive Fasern signifikant erhöht [131]. Letztlich zeigte sich bei der Untersuchung der Aspirin-sensitiven Rhinitis, dass bei diesem Subtyp der chronisch-entzündlichen Rhinitis nur eine erhöhte VIP-Innervationsdichte vorlag, während die Zahlen für Substanz P-, NPY- und CGRP-positive Fasern nicht variierten [132].
Letztlich zeigen diese für die verschiedenen Erkrankungen gewonnenen Daten, dass es innerhalb der verschiedenen Rhinitisformen wesentliche Unterschiede bezüglich der Expression peptiderger Mediatoren in Atemwegsneuronen gibt. Diese Unterschiede weisen darauf hin, dass die Induktion von Neuromediatoren nicht nur als ein reines Epiphänomen entzündlicher Erkrankungen zu betrachten ist, sondern dass es krankheitsspezifische Ursachen der differenziellen Induktion geben muss.

\section{Fazit}

Neben der Bedeutung von VIP für die Regulation der pulmonalen Homöostase unter normalen Bedingungen konnten jüngste Studien zeigen, dass dieser Neuromediator auch bei der Pathophysiologie chronisch-entzündlicher Erkrankungen der unteren und oberen Atemwege sowie bei pulmonaler Hypertonie eine wesentliche Rolle spielt. Dabei ist aufgrund starker bronchodilatorischer, vasodilatorischer und anti-inflammatorischer Effekte auch ein therapeutischer Nutzen des Peptids und seiner synthetischer Agonisten bei Asthma bronchiale, COPD oder pulmonaler Hypertonie in Zukunft denkbar.

\section{Literatur}

${ }^{1}$ Barnes PJ. Asthma as an axon reflex. Lancet 1986; 1: $242-245$

${ }^{2}$ Chung KF, Barnes PJ. Cytokines in asthma. Thorax 1999; 54: 825 - 857

${ }^{3}$ Gozes I, Fridkin M, Brenneman DE. A VIP hybrid antagonist: from developmental neurobiology to clinical applications. Cell Mol Neurobiol 1995; 15: 675-687

${ }^{4}$ Eynott PR, Groneberg DA, Caramori G et al. Role of nitric oxide in allergic inflammation and bronchial hyperresponsiveness. Eur J Pharmacol 2002; 452: $123-133$

${ }^{5}$ Eynott PR, Paavolainen N, Groneberg DA et al. Role of nitric oxide in chronic allergen-induced airway cell proliferation and inflammation. J Pharmacol Exp Ther 2003; 304: 22 - 29

${ }^{6}$ Joos GF, Pauwels RA. Tachykinin receptor antagonists: potential in airways diseases. Curr Opin Pharmacol 2001; 1: 235 - 241

${ }^{7}$ Barnes PJ, Chung KF, Page CP. Inflammatory mediators of asthma: an update. Pharmacol Rev 1998; 50: 515 - 596

${ }^{8}$ Richardson JD, Vasko MR. Cellular mechanisms of neurogenic inflammation. J Pharmacol Exp Ther 2002; 302: 839-845

${ }^{9}$ Velden VH van der, Hulsmann AR. Autonomic innervation of human airways: structure, function, and pathophysiology in asthma. Neuroimmunomodulation 1999; 6: 145-159

10 Widdicombe JG. Autonomic regulation. i-NANC/e-NANC. Am J Respir Crit Care Med 1998; 158: S171 - 175

${ }^{11}$ Linden A. NANC neural control of airway smooth muscle tone. Gen Pharmacol 1996; 27: 1109-1121

12 Widdicombe JG. Overview of neural pathways in allergy and asthma. Pulm Pharmacol Ther 2003; 16: $23-30$

13 Belvisi MG. Sensory nerves and airway inflammation: role of A delta and C-fibres. Pulm Pharmacol Ther 2003; 16: $1-7$

${ }^{14}$ Groneberg DA, Springer J, Fischer A. Vasoactive intestinal polypeptide as mediator of asthma. Pulm Pharmacol Ther 2001; 14: 391 - 401

15 Bedoui S, Kawamura N, Straub RH et al. Relevance of neuropeptide Y for the neuroimmune crosstalk. J Neuroimmunol 2003; 134: 1 -11

${ }^{16}$ Fischer A, Folkerts G, Geppetti P et al. Mediators of asthma: nitric oxide. Pulm Pharmacol Ther 2002; 15: 73-81

17 Groneberg DA, Fischer A. Endogenous opioids as mediators of asthma. Pulm Pharmacol Ther 2001; 14: 383-389

18 Goetzl EJ, Voice JK, Shen S et al. Enhanced delayed-type hypersensitivity and diminished immediate-type hypersensitivity in mice lacking the inducible $\operatorname{VPAC}(2)$ receptor for vasoactive intestinal peptide. Proc Natl Acad Sci USA 2001; 98: 13854 - 13859

${ }^{19}$ Fischer TC, Hartmann P, Loser C et al. Abundant expression of vasoactive intestinal polypeptide receptor VPAC2 mRNA in human skin. J Invest Dermatol 2001; 117: $754-756$ 
${ }^{20}$ Said SI, Mutt V. A peptide fraction from lung tissue with prolonged peripheral vasodilator activity. Scand J Clin Lab Invest Suppl 1969; 107: $51-56$

${ }^{21}$ Said SI, Mutt V. Potent peripheral and splanchnic vasodilator peptide from normal gut. Nature 1970; 225: 863-864

${ }^{22}$ Said SI, Mutt V. Polypeptide with broad biological activity: isolation from small intestine. Science 1970; 169: 1217-1218

${ }^{23}$ Gozes I, Nakai H, Byers M et al. Sequential expression in the nervous system of c-myb and VIP genes, located in human chromosomal region 6q24. Somat Cell Mol Genet 1987; 13: 305-313

${ }^{24}$ Gozes I, Brenneman DE. VIP: molecular biology and neurobiological function. Mol Neurobiol 1989; 3: 201-236

${ }^{25}$ Lilly CM, Drazen JM, Shore SA. Peptidase modulation of airway effects of neuropeptides. Proc Soc Exp Biol Med 1993; 203: 388-404

${ }^{26}$ Hachisu M, Hiranuma T, Tani S et al. Enzymatic degradation of helodermin and vasoactive intestinal polypeptide. J Pharmacobiodyn 1991; 14: 126 - 131

${ }^{27}$ Lundberg JM, Fahrenkrug J, Hokfelt T et al. Co-existence of peptide HI (PHI) and VIP in nerves regulating blood flow and bronchial smooth muscle tone in various mammals including man. Peptides 1984; 5 : 593-606

${ }^{28}$ Baraniuk JN, Lundgren JD, Okayama M et al. Vasoactive intestinal peptide in human nasal mucosa. J Clin Invest 1990; 86: 825 - 831

${ }^{29}$ Bowden JJ, Gibbins IL. Vasoactive intestinal peptide and neuropeptide $Y$ coexist in non-noradrenergic sympathetic neurons to guinea pig trachea. J Auton Nerv Syst 1992; 38: 1-19

${ }^{30}$ Fischer A, Hoffmann B. Nitric oxide synthase in neurons and nerve fibers of lower airways and in vagal sensory ganglia of man. Correlation with neuropeptides. Am J Respir Crit Care Med 1996; 154: 209-216

${ }^{31}$ Carstairs JR, Barnes PJ. Visualization of vasoactive intestinal peptide receptors in human and guinea pig lung. J Pharmacol Exp Ther 1986; 239: $249-255$

${ }^{32}$ Ishihara T, Shigemoto R, Mori K et al. Functional expression and tissue distribution of a novel receptor for vasoactive intestinal polypeptide. Neuron 1992; 8: $811-819$

${ }^{33}$ Lutz EM, Sheward WJ, West KM et al. The VIP2 receptor: molecular characterisation of a cDNA encoding a novel receptor for vasoactive intestinal peptide. FEBS Lett 1993; 334: 3-8

${ }^{34}$ Ciccarelli E, Vilardaga JP, de Neef P et al. Properties of the VIP-PACAP type II receptor stably expressed in CHO cells. Regul Pept 1994; 54: $397-407$

${ }^{35}$ Rawlings SR, Piuz I, Schlegel W et al. Differential expression of pituitary adenylate cyclase-activating polypeptide/vasoactive intestinal polypeptide receptor subtypes in clonal pituitary somatotrophs and gonadotrophs. Endocrinology 1995; 136: 2088-2098

${ }^{36}$ Harmar AJ, Arimura A, Gozes I et al. International Union of Pharmacology. XVIII. Nomenclature of receptors for vasoactive intestinal peptide and pituitary adenylate cyclase-activating polypeptide. Pharmacol Rev 1998; 50: 265-270

${ }^{37}$ Couvineau A, Rouyer-Fessard C, Darmoul D et al. Human intestinal VIP receptor: cloning and functional expression of two cDNA encoding proteins with different $\mathrm{N}$-terminal domains. Biochem Biophys Res Commun 1994; 200: 769-776

${ }^{38}$ Couvineau A, Rouyer-Fessard C, Maoret JJ et al. Vasoactive intestinal peptide (VIP)1 receptor. Three nonadjacent amino acids are responsible for species selectivity with respect to recognition of peptide histidine isoleucineamide. J Biol Chem 1996; 271: 12795-12800

${ }^{39}$ Sreedharan SP, Patel DR, Huang JX et al. Cloning and functional expression of a human neuroendocrine vasoactive intestinal peptide receptor. Biochem Biophys Res Commun 1993; 193: 546-553

${ }^{40}$ Gourlet P, Vandermeers A, Vertongen P et al. Development of high affinity selective VIP1 receptor agonists. Peptides 1997; 18: 1539-1545

${ }^{41}$ Gourlet $\mathrm{P}$, de Neef P, Cnudde J et al. In vitro properties of a high affinity selective antagonist of the VIP1 receptor. Peptides 1997; 18: $1555-1560$

42 Inagaki N, Yoshida $\mathrm{H}$, Mizuta $\mathrm{M}$ et al. Cloning and functional characterization of a third pituitary adenylate cyclase-activating polypeptide receptor subtype expressed in insulin-secreting cells. Proc Natl Acad Sci U S A 1994; 91: 2679-2683

${ }^{43}$ Usdin TB, Bonner TI, Mezey E. Two receptors for vasoactive intestinal polypeptide with similar specificity and complementary distributions. Endocrinology 1994; 135: 2662-2680
${ }^{44}$ Svoboda M, Tastenoy M, van Rampelbergh J et al. Molecular cloning and functional characterization of a human VIP receptor from SUP-T1 lymphoblasts. Biochem Biophys Res Commun 1994; 205: 1617 - 1624

${ }^{45}$ Wei Y, Mojsov S. Tissue specific expression of different human receptor types for pituitary adenylate cyclase activating polypeptide and vasoactive intestinal polypeptide: implications for their role in human physiology. J Neuroendocrinol 1996; 8: $811-817$

${ }^{46}$ Gourlet P, Vertongen P, Vandermeers A et al. The long-acting vasoactive intestinal polypeptide agonist RO $25-1553$ is highly selective of the VIP2 receptor subclass. Peptides 1997; 18: $403-408$

47 O’Donnell M, Garippa RJ, Rinaldi N et al. Ro 25-1553: a novel, longacting vasoactive intestinal peptide agonist. Part I: In vitro and in vivo bronchodilator studies. J Pharmacol Exp Ther 1994; 270: 1282-1288

${ }^{48}$ O'Donnell M, Garippa RJ, Rinaldi N et al. Ro 25-1553: a novel, longacting vasoactive intestinal peptide agonist. Part II: Effect on in vitro and in vivo models of pulmonary anaphylaxis. J Pharmacol Exp Ther 1994; 270: 1289 - 1294

${ }^{49}$ Tang H, Welton A, Ganea D. Neuropeptide regulation of cytokine expression: effects of VIP and Ro 25-1553. J Interferon Cytokine Res 1995; 15: $993-1003$

${ }^{50}$ Xia M, Sreedharan SP, Bolin DR et al. Novel cyclic peptide agonist of high potency and selectivity for the type II vasoactive intestinal peptide receptor. J Pharmacol Exp Ther 1997; 281: 629-633

${ }^{51}$ Fischer TC, Dinh QT, Peiser C et al. Simultaneous detection of receptor mRNA and ligand protein in human skin tissues. J Cutan Pathol 2002; 29: $65-71$

52 Groneberg DA, Hartmann P, Dinh QT et al. Expression and distribution of vasoactive intestinal polypeptide receptor VPAC(2) mRNA in human airways. Lab Invest 2001; 81: 749-755

${ }^{53}$ Busto R, Prieto JC, Bodega G et al. Immunohistochemical localization and distribution of VIP/PACAP receptors in human lung. Peptides 2000; 21: 265-269

54 Peiser C, Springer J, Groneberg DA et al. Leptin receptor expression in nodose ganglion cells projecting to the rat gastric fundus. Neurosci Lett 2002; 320: $41-44$

${ }^{55}$ Fischer A, Kummer W, Couraud JY et al. Immunohistochemical localization of receptors for vasoactive intestinal peptide and substance $P$ in human trachea. Lab Invest 1992; 67: 387-393

${ }^{56}$ Voice JK, Dorsam G, Lee H et al. Allergic diathesis in transgenic mice with constitutive T cell expression of inducible vasoactive intestinal peptide receptor. FASEB J 2001; 15: 2489-2496

57 Palmer JB, Cuss FM, Barnes PJ. VIP and PHM and their role in nonadrenergic inhibitory responses in isolated human airways. J Appl Physiol 1986; 61: 1322-1328

58 Altiere RJ, Diamond L. Comparison of vasoactive intestinal peptide and isoproterenol relaxant effects in isolated cat airways. J Appl Physiol 1984; 56: $986-992$

${ }^{59}$ Hand JM, Laravuso RB, Will JA. Relaxation of isolated guinea pig trachea, bronchi and pulmonary arteries produced by vasoactive intestinal peptide (VIP). Eur J Pharmacol 1984; 98: 279-284

${ }^{60}$ Saga T, Said SI. Vasoactive intestinal peptide relaxes isolated strips of human bronchus, pulmonary artery, and lung parenchyma. Trans Assoc Am Physicians 1984; 97: 304 - 310

${ }^{61}$ Diamond L, Szarek JL, Gillespie MN et al. In vivo bronchodilator activity of vasoactive intestinal peptide in the cat. Am Rev Respir Dis 1983; 128: 827-832

62 Hamasaki Y, Saga T, Mojarad M et al. Vasoactive intestinal peptide counteracts leukotriene D4-induced contractions of guinea pig trachea, lung, and pulmonary artery. Trans Assoc Am Physicians 1983; 96: $406-411$

63 Boomsma JD, Said SI. The role of neuropeptides in asthma. Chest 1992; 101: 389S-392S

${ }^{64}$ Cox CP, Lerner MR, Wells JJ et al. Inhaled vasoactive intestinal peptide (VIP) prevents bronchoconstriction induced by inhaled histamine. Am Rev Respir Dis 1983; 127: A249

${ }^{65}$ Bundgaard A, Enehjelm SD, Aggestrup S. Pretreatment of exercise-induced asthma with inhaled vasoactive intestinal peptide (VIP). Eur J Respir Dis Suppl 1983; 128: 427-429

${ }^{66}$ Altiere RJ, Kung M, Diamond L. Comparative effects of inhaled isoproterenol and vasoactive intestinal peptide on histamine-induced bronchoconstriction in human subjects. Chest 1984; 86: 153-154

${ }^{67}$ Morice A, Unwin RJ, Sever PS. Vasoactive intestinal peptide causes bronchodilatation and protects against histamine-induced bronchoconstriction in asthmatic subjects. Lancet 1983; 2: 1225-1227 
${ }^{68}$ Groneberg DA, Nickolaus M, Springer J et al. Localization of the peptide transporter PEPT2 in the lung: implications for pulmonary oligopeptide uptake. Am J Pathol 2001; 158: 707 - 714

${ }^{69}$ Groneberg DA, Eynott PR, Doring F et al. Distribution and function of the peptide transporter PEPT2 in normal and cystic fibrosis human lung. Thorax 2002; 57: 55-60

${ }^{70}$ Groneberg DA. Expression, Lokalisation und funktionelle Aspekte des Peptidtransporters PEPT2 im gesunden Atemtrakt und bei Mukoviszidose. Pneumologie 2003; 57: 104-105

${ }^{71}$ Groneberg DA, Doring F, Nickolaus $\mathrm{M}$ et al. Expression of PEPT2 peptide transporter mRNA and protein in glial cells of rat dorsal root ganglia. Neurosci Lett 2001; 304: 181 - 184

72 Sharaf H, Said S. I. Tracheal relaxant response to vasoactive intestinal peptide (VIP): influence of airway epithelium and peptidases (abstract). FASEB J 1993; 7: A686

${ }^{73}$ Bolin DR, Cottrell J, Garippa R et al. Structure-activity studies of vasoactive intestinal peptide (VIP): cyclic disulfide analogs. Int J Pept Protein Res 1993; 41: 124-132

${ }^{74}$ Ito O, Tachibana S. Vasoactive intestinal polypeptide precursors have highly potent bronchodilatory activity. Peptides 1991; 12: 131-137

${ }^{75}$ Schmidt DT, Ruhlmann E, Waldeck B et al. The effect of the vasoactive intestinal polypeptide agonist Ro 25-1553 on induced tone in isolated human airways and pulmonary artery. Naunyn Schmiedebergs Arch Pharmacol 2001; 364: 314-320

${ }^{76}$ Lung MA, Widdicombe JG. Lung reflexes and nasal vascular resistance in the anaesthetized dog. J Physiol (Lond) 1987; 386: 465-474

${ }^{77}$ Lundberg JM, Anggard A, Emson P et al. Vasoactive intestinal polypeptide and cholinergic mechanisms in cat nasal mucosa: studies on choline acetyltransferase and release of vasoactive intestinal polypeptide. Proc Natl Acad Sci U S A 1981; 78: 5255-5259

${ }^{78}$ Laitinen LA, Laitinen A, Salonen RO et al. Vascular actions of airway neuropeptides. Am Rev Respir Dis 1987; 136: S59-64

${ }^{79}$ Hamasaki Y, Mojarad M, Said SI. Relaxant action of VIP on cat pulmonary artery: comparison with acetylcholine, isoproterenol, and PGE1. J Appl Physiol 1983; 54: 1607-1611

${ }^{80}$ Nandiwada PA, Kadowitz PJ, Said SI et al. Pulmonary vasodilator responses to vasoactive intestinal peptide in the cat. J Appl Physiol 1985; 58 : 1723 - 1728

81 Obara H, Kusunoki M, Mori M et al. The effects of various peptides on the isolated pulmonary artery. Peptides 1989; 10: $241-243$

${ }^{82}$ Matran R, Alving K, Martling CR et al. Effects of neuropeptides and capsaicin on tracheobronchial blood flow of the pig. Acta Physiol Scand 1989; 135 : $335-342$

${ }^{83}$ Greenberg B, Rhoden K, Barnes PJ. Relaxant effects of vasoactive intestinal peptide and peptide histidine isoleucine in human and bovine pulmonary arteries. Blood Vessels 1987; 24: 45-50

${ }^{84}$ Barnes PJ, Cadieux A, Carstairs JR et al. Vasoactive intestinal peptide in bovine pulmonary artery: localisation, function and receptor autoradiography. Br J Pharmacol 1986; 89: 157-162

${ }^{85}$ Groneberg DA, Peiser C, Dinh QT et al. Distribution of respiratory mucin proteins in human nasal mucosa. Laryngoscope 2003; 113: 520-524

${ }^{86}$ Groneberg DA, Eynott PR, Oates T et al. Expression of MUC5AC and MUC5B mucins in normal and cystic fibrosis lung. Respir Med 2002; 96: $81-86$

${ }^{87}$ Groneberg DA, Eynott PR, Lim S et al. Expression of respiratory mucins in fatal status asthmaticus and mild asthma. Histopathology 2002; 40: 367-373

88 Groneberg DA, Wagner U, Chung KF. Mucus and fatal asthma. Am J Med 2004; 116: 66-67

89 Dey RD, Shannon Jr WA, Said SI. Localization of VIP-immunoreactive nerves in airways and pulmonary vessels of dogs, cat, and human subjects. Cell Tissue Res 1981; 220: 231 - 238

90 Peatfield AC, Barnes PJ, Bratcher C et al. Vasoactive intestinal peptide stimulates tracheal submucosal gland secretion in ferret. Am Rev Respir Dis 1983; 128: 89-93

${ }^{91}$ Wagner U, Bredenbroker D, Storm B et al. Effects of VIP and related peptides on airway mucus secretion from isolated rat trachea. Peptides 1998; 19: 241 - 245

92 Webber SE, Widdicombe JG. The effect of vasoactive intestinal peptide on smooth muscle tone and mucus secretion from the ferret trachea. Br J Pharmacol 1987; 91: 139-148

${ }^{93}$ Shimura S, Sasaki T, Ikeda K et al. VIP augments cholinergic-induced glycoconjugate secretion in tracheal submucosal glands. J Appl Physiol 1988; 65: $2537-2544$
${ }^{94}$ Nathanson I, Widdicombe JH, Barnes PJ. Effect of vasoactive intestinal peptide on ion transport across dog tracheal epithelium. J Appl Physiol 1983; 55: $1844-1848$

${ }^{95}$ Richardson PS, Webber SE. The control of mucous secretion in the airways by peptidergic mechanisms. Am Rev Respir Dis 1987; 136: S72- 76

${ }^{96}$ Sakai N, Tamaoki J, Kobayashi $\mathrm{K}$ et al. Vasoactive intestinal peptide stimulates ciliary motility in rabbit tracheal epithelium: modulation by neutral endopeptidase. Regul Pept 1991; 34: 33-41

${ }^{97}$ Coles SJ, Said SI, Reid LM. Inhibition by vasoactive intestinal peptide of glycoconjugate and lysozyme secretion by human airways in vitro. Am Rev Respir Dis 1981; 124: 531-536

98 Goetzl EJ, Pankhaniya RR, Gaufo GO et al. Selectivity of effects of vasoactive intestinal peptide on macrophages and lymphocytes in compartmental immune responses. Ann N Y Acad Sci 1998; 840: $540-550$

${ }^{99}$ Said SI, Dickman KG. Pathways of inflammation and cell death in the lung: modulation by vasoactive intestinal peptide. Regul Pept 2000; 93: $21-29$

${ }^{100}$ Nio DA, Moylan RN, Roche JK. Modulation of T lymphocyte function by neuropeptides. Evidence for their role as local immunoregulatory elements. J Immunol 1993; 150: 5281-5288

101 Undem BJ, Dick EC, Buckner CK. Inhibition by vasoactive intestinal peptide of antigen-induced histamine release from guinea-pig minced lung. Eur J Pharmacol 1983; 88: 247-250

102 O'Dorisio MS, Shannon BT, Fleshman DJ et al. Identification of high affinity receptors for vasoactive intestinal peptide on human lymphocytes of B cell lineage. J Immunol 1989; 142: 3533-3536

103 Berisha H, Foda H, Sakakibara $\mathrm{H}$ et al. Vasoactive intestinal peptide prevents lung injury due to xanthine/xanthine oxidase. Am J Physiol 1990; 259: L151 - 155

104 Misra BR, Misra HP. Vasoactive intestinal peptide, a singlet oxygen quencher. J Biol Chem 1990; 265: 15371 - 15374

105 Leceta J, Gomariz RP, Martinez C et al. Receptors and transcriptional factors involved in the anti-inflammatory activity of VIP and PACAP. Ann N Y Acad Sci 2000; 921: $92-102$

106 Delgado M, Abad C, Martinez C et al. Vasoactive intestinal peptide prevents experimental arthritis by downregulating both autoimmune and inflammatory components of the disease. Nat Med 2001; 7: $563-568$

107 Groneberg DA, Welker P, Fischer TC et al. Down-regulation of vasoactive intestinal polypeptide receptor expression in atopic dermatitis. J Allergy Clin Immunol 2003; 111: 1099-1105

108 Ollerenshaw S, Jarvis D, Woolcock A et al. Absence of immunoreactive vasoactive intestinal polypeptide in tissue from the lungs of patients with asthma. N Engl J Med 1989; 320: 1244-1248

${ }^{109}$ Howarth PH, Djukanovic R, Wilson JW et al. Mucosal nerves in endobronchial biopsies in asthma and non-asthma. Int Arch Allergy Appl Immunol 1991; 94: 330-333

${ }^{110}$ Howarth PH, Springall DR, Redington AE et al. Neuropeptide-containing nerves in endobronchial biopsies from asthmatic and nonasthmatic subjects. Am J Respir Cell Mol Biol 1995; 13: 288-296

111 Chanez P, Springall D, Vignola AM et al. Bronchial mucosal immunoreactivity of sensory neuropeptides in severe airway diseases. Am J Respir Crit Care Med 1998; 158: 985-990

112 Kaltreider HB, Ichikawa S, Byrd PK et al. Upregulation of neuropeptides and neuropeptide receptors in a murine model of immune inflammation in lung parenchyma. Am J Respir Cell Mol Biol 1997; 16: $133-144$

113 Barnes PJ. Vasoactive intestinal peptide and asthma. N Engl J Med 1989; 321: 1128 - 1129

114 Wenzel SE, Fowler AAd, Schwartz LB. Activation of pulmonary mast cells by bronchoalveolar allergen challenge. In vivo release of histamine and tryptase in atopic subjects with and without asthma. Am Rev Respir Dis 1988; 137: 1002 - 1008

115 Paul S, Said SI, Thompson AB et al. Characterization of autoantibodies to vasoactive intestinal peptide in asthma. J Neuroimmunol 1989; 23: $133-142$

116 Ohzeki T, Ishitani N, Hanaki K et al. Responses of plasma vasoactive intestinal polypeptide to methacholine and exercise loading in children and adolescents with bronchial asthma. Pediatr Allergy Immunol $1993 \cdot 4 \cdot 26-29$

117 Cardell LO, Uddman R, Edvinsson L. Low plasma concentrations of VIP and elevated levels of other neuropeptides during exacerbations of asthma. Eur Respir J 1994; 7: 2169-2173 
118 Palmer JB, Cuss FM, Warren JB et al. Effect of infused vasoactive intestinal peptide on airway function in normal subjects. Thorax 1986; 41: 663-666

119 Barnes PJ, Dixon CM. The effect of inhaled vasoactive intestinal peptide on bronchial reactivity to histamine in humans. Am Rev Respir Dis 1984; 130: 162 - 166

${ }^{120}$ Linden A, Hansson L, Andersson A et al. Bronchodilation by an inhaled $\operatorname{VPAC}(2)$ receptor agonist in patients with stable asthma. Thorax 2003; 58: $217-221$

${ }^{121}$ Runo JR, Loyd JE. Primary pulmonary hypertension. Lancet 2003; 361: $1533-1544$

122 Olschewski H, Ghofrani A, Wiedemann R et al. Pulmonaler Hochdruck. Internist (Berl) 2002; 43: 1498, 1501 - 1509

${ }^{123}$ Olschewski H, Seeger W. Behandlung der Pulmonal Arteriellen Hypertonie. Pneumologie 2000; 54: 222-224

${ }^{124}$ Ghofrani HA, Olschewski H, Seeger W et al. Sildenafil zur Therapie der schweren pulmonalen Hypertonie und des beginnenden Rechtsherzversagens. Pneumologie 2002; 56: 665-672

${ }^{125}$ Ghofrani HA, Rose F, Schermuly RT et al. Oral sildenafil as long-term adjunct therapy to inhaled iloprost in severe pulmonary arterial hypertension. J Am Coll Cardiol 2003; 42: 158 - 164
${ }^{126}$ Rich S, Kaufmann E, Levy PS. The effect of high doses of calcium-channel blockers on survival in primary pulmonary hypertension. $\mathrm{N}$ Engl J Med 1992; 327: 76-81

${ }^{127}$ Keith IM. The role of endogenous lung neuropeptides in regulation of the pulmonary circulation. Physiol Res 2000; 49: 519-537

${ }^{128}$ Petkov V, Mosgoeller W, Ziesche R et al. Vasoactive intestinal peptide as a new drug for treatment of primary pulmonary hypertension. J Clin Invest 2003; 111: 1339-1346

129 Jones AS. Non-allergic perennial rhinitis. Biomed Pharmacother 1988; 42: 499-503

130 Groneberg DA, Heppt W, Cryer A et al. Toxic rhinitis-induced changes of human nasal mucosa innervation. Toxicol Pathol 2003; 31: $326-331$

${ }^{131}$ Heppt W, Peiser C, Cryer A et al. Innervation of human nasal mucosa in environmentally triggered hyperreflectoric rhinitis. J Occup Environ Med 2002; 44: 924-929

132 Groneberg DA, Heppt W, Welker P et al. Aspirin-sensitive rhinitis associated changes in upper airway innervation. Eur Respir J 2003; 22: 986-991

\section{Buchbesprechung}

\section{Tuberkulose als Berufskrankheit. Ein Leitfaden zur Begutachtung}

A. Nienhaus, S. Brandenburg, H. Teschler

Landsberg: Ecomed Medizin, 2003. 209 S. ISBN 3-609-16231-7

Bei einem langjährig als Gutachter tätigen Pneumologen weckt der Titel des zu besprechenden Buches „Tuberkulose als Berufskrankheit" sofort Interesse. Man erinnert sich an schwierige Diskussionen mit den Beratungsärzten der Berufsgenossenschaften, fast immer die für Gesundheitsdienst und Wohlfahrtspflege. Wie schwer ist es immer, die berufsbedingte Erkrankung als wahrscheinlich zu deklarieren, wenn die BGW anderer Meinung ist. Die Herausgeber erweisen sich als Team mit dem Chef der BGW BV Hamburg, einem Arzt aus dem gleichen Hause und einem erfahrenen Gutachter, der sich dazu noch intensiv um die Weiterbildung der Pneumologen im Gutachtenwesen kümmert. Die zahlreichen Autoren bringen als Epidemiologen, als Mitglieder des DZK, Praktiker im Alltag der Begutachtung und als Forscher im Bereich der Infektionswege ihr Wissen ein.

Die ersten Kapitel sind der Geschichte der Tuberkulose als Berufskrankheit und der Epidemiologie gewidmet sowie der Praxis der Meldeverfahren, die oft genug zu wünschen übrig lässt. Die Voraussetzungen der BK-Anerkennung und die Voraussetzungen für eine Entschädigung werden von juristischer Warte besprochen. Ausgesprochene Relevanz hat das Kapitel über die Anzeigepflicht der Tuberkulinkonversion bei Angehörigen im Gesundheitswesen und die zu ziehenden Konsequenzen.

Ein eigenes Kapitel ist der Wertigkeit der neuen bildgebenden Verfahren gewidmet, wobei dem CT die überragende Bedeutung bei der zuverlässigen Einordnung der Lungen-, Mediastinal- und Pleuraveränderungen zukommt. Wichtig ist die ausführliche
Darstellung der Strahlenbelastung der verschiedenen Röntgenuntersuchungen, damit eine strenge Indikationsstellung geübt wird.

Für die meisten neu, und aufgrund der angewandten Technik, auch erst einmal nicht so einfach zu verstehen, dürften die Kapitel über die molekularbiologische Charakterisierung des M. tuberkulosis sein. Sie ermöglicht den zweifelsfreien Nachweis von Infektionsketten und hat dazu beigetragen, dass wir heute sehr viel häufiger von Superinfektionen als Ursache von aktiven Tuberkulosen ausgehen müssen. Das früher geltende Dogma „früher positiver Tuberkulintest oder alte spezifische Narben in der Voraufnahme schließen eine frische beruflich erworbene Tuberkulose aus" kann in einer Reihe von Fällen widerlegt werden. Leider zeigen die Vergleiche internationaler Daten noch kein einheitliches Bild. Trotzdem geht aus den Diskussionen in dem Workshop, aus dem das Buch als Zusammenfassung stammt, eine Empfehlung zur Beweiserleichterung für eine Reihe von Ansteckungs- und Erkrankungsfällen hervor, die in Zukunft eine raschere Bearbeitung von beruflich erworbenen Tuberkulosen erwarten lassen, insbesondere auch für die eigene Berufsgruppe.

Das Buch liest sich trotz der Vielzahl der Autoren leicht, kann aber ebenso als Nachschlagewerk für die Argumentation bei der eigenen gutachterlichen Arbeit dienen. Da offensichtlich noch Bewegung in der Gesamtproblematik steckt und insbesondere die Identifikation von Infektionspfaden durch Erfassung einer größeren Zahl von Tuberkulosestämmen zu erwarten steht, ist dem Buch eine rasche Überarbeitung auf dem Boden neuer Daten zu wünschen. Bei einem Buch mit Werkstattcharakter und bei der Aktivität der Herausgeber kann man diesbezüglich guter Hoffnung sein.

H. Steveling, Essen 